$R M x A C, \mathbf{5 3}, 215-224(2021)$

(c) 2021: Instituto de Astronomía, Universidad Nacional Autónoma de México

https://doi.org/10.22201/ia.14052059p.2021.53.41

\title{
STUDY OF ENERGETIC TRANSIENTS USING TOOLS LIKE MESA \& SNEC, MOSFIT AND SNCOSMO
}

\author{
Amar Aryan ${ }^{1,2}$, Shashi B. Pandey ${ }^{1,2}$, Amit Kumar ${ }^{1,3}$, Rahul Gupta ${ }^{1,2}$, Alberto J. Castro-Tirado ${ }^{4,5}$, \\ and S. N. Tiwari ${ }^{2}$ \\ RESUMEN
}

Exploramos el estudio de fuentes transitorias de alta energía, incluyendo las supernovas originadas por el colapso del núcleo de la estrella progenitora, utilizando varias herramientas de análisis disponibles públicamente como MESA \& SNEC, MOSFiT y SNCOSMO. Usamos MESA para trazar la evolución de una estrella de secuencia principal de edad cero y masa $\left(M_{z a m s}\right)$ de $24 \mathrm{M}_{\odot}$ hasta el inicio del colapso del núcleo. Luego exploramos este modelo usando códigos de explosión disponibles en abierto, STELLA \& SNEC y obtuvimos varios parámetros observables como luminosidad bolométrica y velocidades fotosféricas, etc. También usamos MOSFiT para modelar la curva de luz de una supernova de tipo Ic, SN1999ex y obtuvimos varios parámetros f́sicos. SNCOSMO se utiliza para el ajuste de plantillas de varias supernovas mediante la variación de varios parámetros, como desplazamiento al rojo, mapa de polvo, factor de estiramiento de la curva de luz, época de explosión de la supernova, etc.

\section{ABSTRACT}

We explore the study of energetic transients including core-collapse supernovae using various publicly available analysis tools like MESA \& SNEC, MOSFiT and SNCOSMO. We used MESA to evolve a star having zero age main sequence mass $\left(M_{z a m s}\right)$ of $24 \mathrm{M}_{\odot}$ until the onset of core-collapse. Then we exploded this model using openly available explosion codes, STELLA \& SNEC and obatined various observable parameters such as bolometric luminosity and photospheric velocities etc. We also used MOSFiT to model the light curve of a type Ic supernova, SN1999ex and obtained various physical parameters. SNCOSMO is used for template fitting of various supernovae by varying various parameters such as red shift, dust map, stretch factor of light curve, explosion epoch of supernova etc.

Key Words: methods: data analysis - supernovae: general

\section{INTRODUCTION}

Transient astrophysical phenomena are the events that occur on a very short-timescale, ranging from a fraction of seconds to weeks or year. Typically, these are extreme and short-lived events that are associated with the partial or complete destruction of an astrophysical object. These events are extremely powerful and can show emission at all or specific wavelengths which can be either electromagnetic waves or gravitational waves. Due to extreme energy emission associated with these events, they can ap-

\footnotetext{
${ }^{1}$ Aryabhatta Research Institute of observational sciencES (ARIES),Manora peak, Nainital, Uttarakhand, India, PIN : 263001 (amar@aries.res.in).

${ }^{2}$ Deen Dayal Upadhyaya Gorakhpur University, Gorakhpur, Uttarpradesh, India, PIN : 273009

${ }^{3}$ Pt. Ravishankar Shukla University, Raipur, Chhattisgarh, India, PIN : 492010

${ }^{4}$ Instituto de Astrofísica de Andalucía (IAA-CSIC), Glorieta de la Astronomía s/n, E-18008, Granada, Spain.

${ }^{5}$ Unidad Asociada Departamento de Ingeniería de Sistemas y Automática, E.T.S. de Ingenieros Industriales, Universidad de Málaga, Spain.
}

pear very bright and hence can be seen from large cosmological distances ${ }^{6}$. Supernovae(SNe), Novae, Dwarf Nova outbursts, Gamma-Ray Bursts (GRBs), Tidal disruption events etc. fall under transients category. In this paper, we are mainly focused on SNe. $\mathrm{SNe}$ are energetic transients that occur during the last evolutionary stages of stars. There exist various classes of SNe. These are mainly classified on the basis of presence or absence of Hydrogen features in their spectra (Filippenko et al. 1997). Those, which show presence of strong Hydrogen features in their spectra are classified as type II SNe while those, which do not, are type I SNe. SNe are further divided into sub-types on the basis of presence or absence of various elements other then Hydrogen such as Helium and Silicon (eg. type Ib and type Ic etc.) and also on the shape of their light curves(Smartt 2009). Type IIP are the SNe which show plateau in their light curves while type IIL SNe shoe a linear decay

\footnotetext{
${ }^{6}$ www.nature.com/subjects/transient-astrophysicalphenomena
} 
after peak brightness in their light curves. Transients have special place in the field of astronomy and astrophysics. These are helpful in unveiling the hidden mysteries of universe. Particularly, SNe shed light on how elements came to be formed, and also on cosmic rays which are accelerating subatomic particle in space. SNe are responsible for the enrichment of the universe. They spread elements such as Oxygen, Nitrogen, Silicon, Nickel, Cobalt, Iron and few other lighter elements in space. Famous type Ia SNe act as standard candles of universe. To establish the properties of massive stars exploding as $\mathrm{SNe}$ is a very difficult and challenging task. The electromagnetic emission that originates during the first minutes to hours after the emergence of the shock from the stellar surface serves extremely important source of information about the final evolution and structure of the exploding star (Bersten et al. 2018), which are among the most challenging task in the field of transients. But, to get such early and serendipitous discoveries, we need to be extremely lucky. In this scenario, the role of robotic telescopes is very significant. The Burst Observer and Optical Transient Exploring System (BOOTES) is one such attempt. The first two BOOTES stations were located in Spain. These two stations included medium size robotic telescopes with CCD cameras at the Cassegrain focus as well as all-sky cameras (Castro-Tirado A. J. 2010). Currently, there are five BOOTES stations, which are located in Spain (BOOTES-1 and BOOTES2), New Zealand (BOOTES-3), China (BOOTES5) and Mexico (BOOTES-5). The primary goal of the BOOTES network is to quickly observe various transient events within few seconds or minutes of being detected by scientific satellites. Besides these prompt optical follow-up observations, this network also responsible for the discovery of various objects such as variable stars, novae and $\mathrm{SNe}$ etc. In case a $\mathrm{SN}$ is discovered in its extreme early phase, such interesting objects can be followed for longer times. Such observations are extremely useful in understating the SN progenitors. While the robotic telescopes are important for prompt discovery and initial follow of many transients, the role of ground based optical telescopes becomes very important for the late follow up of these transients. Few famous large ground based telescopes in earth currently are Large Binocular Telescope (LBT ${ }^{7}$ ), having a combined diameter of around $11.9 \mathrm{~m}$, Gran Telescopio Canarias $\left(\mathrm{GTC}^{8}\right)$, which has a diameter of $10.4 \mathrm{~m}$,

\footnotetext{
${ }^{7}$ https://www.lbto.org/

${ }^{8} \mathrm{http}: / /$ www.gtc.iac.es/
}

Hobby-Eberly Telescope $\left(\mathrm{HET}^{9}\right.$, having a diameter of $10 \mathrm{~m}$, two big telescopes having diameters of $10 \mathrm{~m}$ at Keck observatory ${ }^{10}$ and The Southern African Large Telescope (SALT ${ }^{11}$ ), having an effective diameter of $9.2 \mathrm{~m}$. There are many other ground based optical telescopes currently throughout the globe having relatively smaller diameters. These telescopes are useful for long follow up of optical transients such as novae, dwarf novae, $\mathrm{SNe}$, late time optical after-glow of GRBs. Shashi (2016) explains the use of $3.6 \mathrm{~m}$ Devasthal Optical Telescope (DOT ${ }^{12}$ ) situated at Aryabhatta research institute of observational sciences (ARIES ${ }^{13}$ ), Nainital, India. Fig.1 shows two R-band images of a famous supernova (SN), SN2016gkg taken at an interval of more than one year. We could detect the SN signature even after more than one year. The images are taken with $4 \mathrm{k} \mathrm{X} 4 \mathrm{k}$ imager mounted on $3.6 \mathrm{~m}$ DOT, having a field of view of 6.5' X 6.5' with individual exposures of 200 s and 300 s respectively.

Besides all these observational studies, there are some publicly available tools such as the Modules for Experiments in Stellar Astrophysics (MESA) (Paxton et al. 2011, 2013, 2015, 2018), that can be to reasonably model the progenitor of various SNe. Further, the output of MESA, in appropriate format can be used as input to various openly available explosion code such as STELLA (a part of MESA) and SuperNova Explosioin Code (SNEC) (Morozova et al. 2015), which provide various observable parameters such as bolometric luminosity and photospheric velocities etc. We used a python based openly available tool, Modular Open Source Fitter for Transients(MOSFiT) (Guillochon et al. 2018) to perform light curve fitting and robustly estimating various SN parameters such as the explosion epoch, eject mass, temperature etc. We also used SNCOSMO for template fittings of SNe light curves. The template of $\mathrm{SNe}$ of various types are already available in SNCOSMO (Barbary et al. 2016). We can fit the light curves of any particular supernova (SN) with the available template by varying various parameters such as red shift of SN, explosion date, stretch factor etc.

We have divided our present analysis in various sections. In $\S 2$, we discuss the methods of implying various SN analysis tools briefly. In section $\S 3$, we discuss the outcomes that are obtained using the

\footnotetext{
${ }^{9}$ https://mcdonaldobservatory.org/research/telescopes/HET

${ }^{10}$ http://www.keckobservatory.org/

${ }^{11}$ https://www.salt.ac.za/

${ }^{12}$ https://www.aries.res.in/dot/index.html

${ }^{13}$ https://www.aries.res.in/
} 


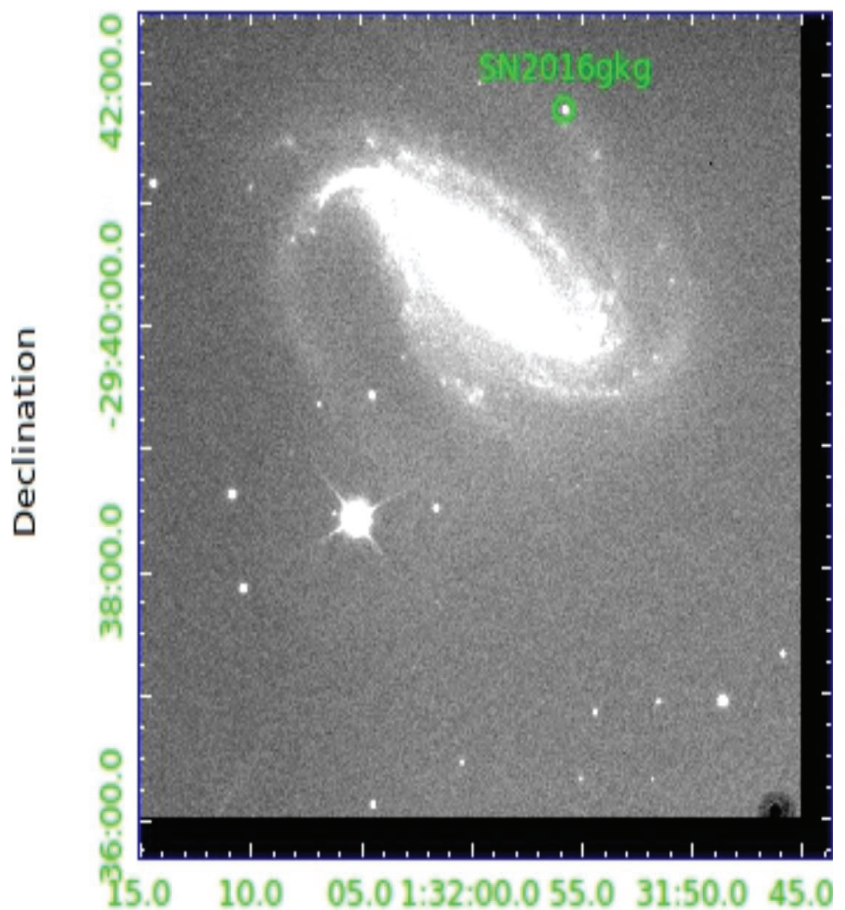

Right ascension

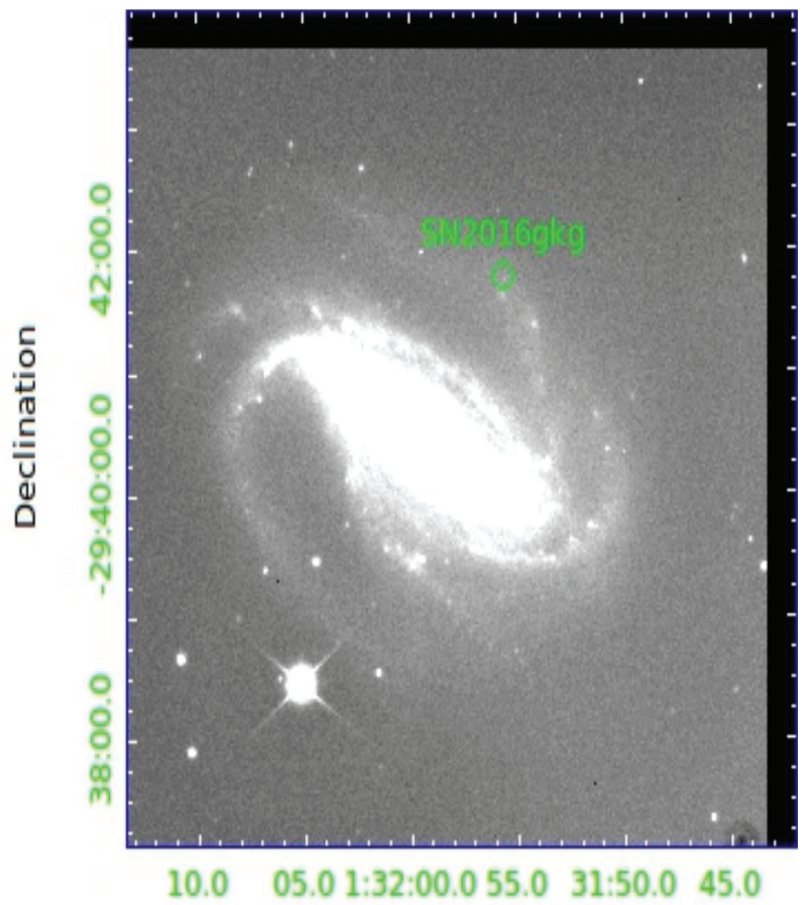

Right ascension

Fig. 1. Images of SN2016gkg taken from 3.6m DOT in R-band. (a) The left panel shows the SN image along with its host galaxy taken on $03^{\text {rd }}$ November 2016, with $4 \mathrm{k} \mathrm{X} 4 \mathrm{k}$ imager mounted on $3.6 \mathrm{~m}$ DOT in $2 \times 2$ binning mode. (b) The right panel shows the same SN image after around 384 days on $22^{\text {nd }}$ March 2017 taken with same instrument mounted on $3.6 \mathrm{~m}$ DOT in nearly similar configuration. In the second image, we can clearly see the SN signature

above mentioned SN analysis tools. Finally, we summarize and conclude our analysis in $\S 4$.

\section{DESCRIPTION OF TOOLS}

In this section, we demonstrate the powerfulness of various transient analysis tools. We have shown here the methods of implementation of these publicly available tools to perform robust analysis.

\subsection{MESA}

MESA is an openly available one dimensional stellar evolution code which can be downloaded by anyone from its website ${ }^{14}$. It can perform the evolution of various stars having different physical scenarios. Separate modules are available for performing different kind of evolutions. Starting from the evolution of a $1 \mathrm{M}_{\odot}$ star, it can perform accretion onto a neutron star and this code is also capable of simulating explosive nucleosynthesis. Here in this particular project, we have taken a star having $\mathrm{M}_{\text {zams }}$ of $24 \mathrm{M}_{\odot}$. We evolved this star up to the stage, where it can undergo core-collapse. For the same purpose,

\footnotetext{
${ }^{14}$ mesa.sourceforge.net
}

MESA version 11701 is used. For simplicity, rotation aspects are not considered. We have used the initial metallicity of $\mathrm{Z}=0.02$, and modeled convection using the mixing theory of (Henyey et al. 1965) by adopting the Ledoux criterion. For modelling the convective overshooting, we have used the diffusive approach of Herwig (2000) with $\mathrm{f}=0.01$ and $\mathrm{f}_{0}=$ 0.004 for all the convective core and shells. After the successful evolution of the star through various stages of its life, it finally reaches to the stage where it can undergo core-collapse. The outputs of final stage evolution through MESA were then used as input to various publicly available codes to simulate the explosion numerically.

STELLA is a hydro dynamical code that can be used to simulate energetic core-collapse SN explosions. It solves the radiative transfer equations in the intensity momentum approximation in each frequency bin. In MESA, STELLA is run using 40 frequency groups, which is enough to produce spectral energy distribution, but only this many number of frequency group is not sufficient to produce spectra. Here the opacity is computed based on over 153,000 spectral lines from (Kurucz R. L. \& Bell 


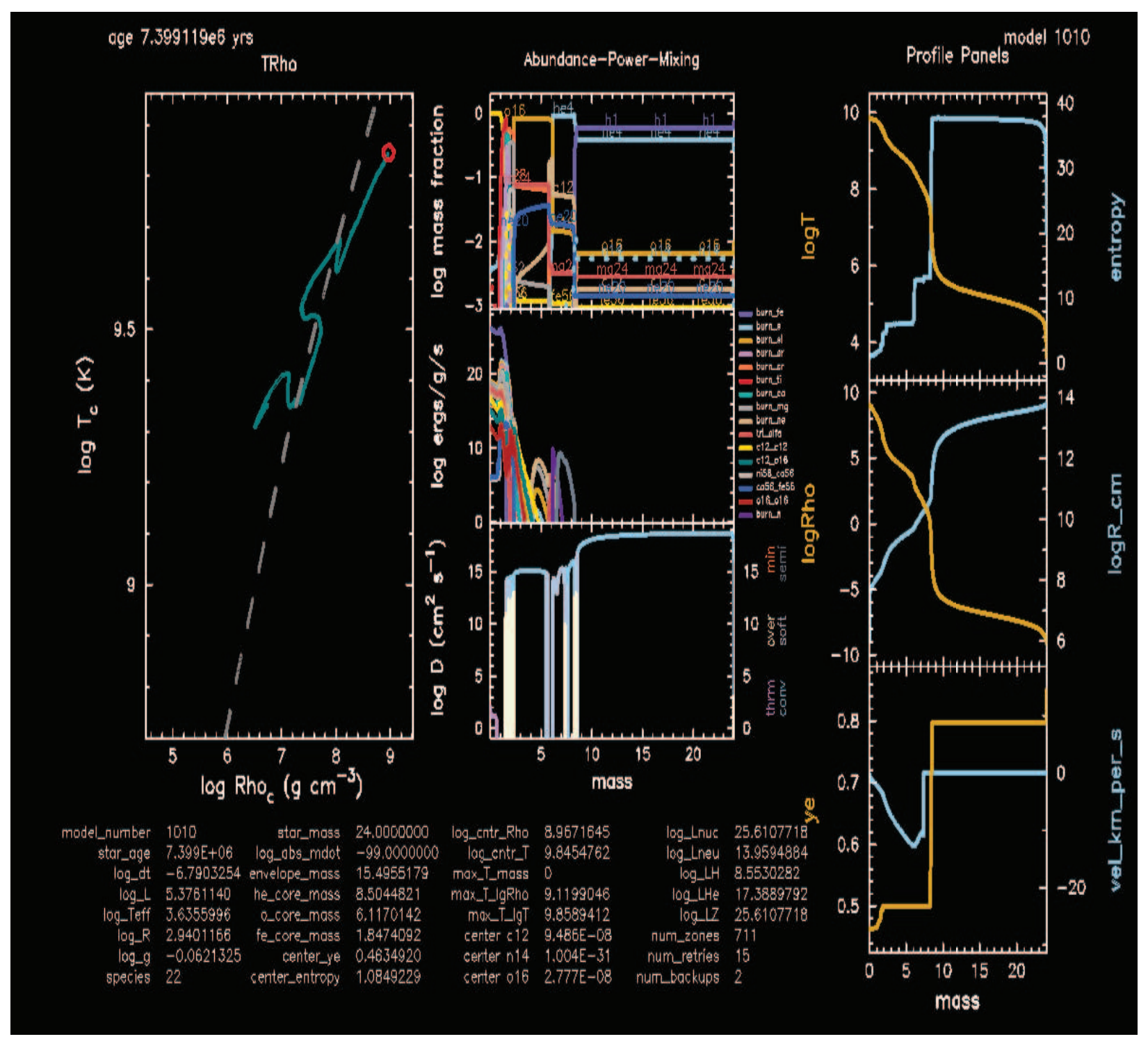

Fig. 2. A glimpse of our model star's condition after it has finished burning Si in its core and tending to reach a core temperature, $\log \mathrm{T}$ of $9.9 \mathrm{~K}$.

B., 1995) and (Verner, Verner \& Ferland 1996). To compute the line opacity by taking into account the effect of high velocity gradients, the expansion opacity formalism from (Eastman \& Pinto 1993) is used. Here the photo-ionization, free-free absorption and electron scattering are also the sources of opacity that are included. Local thermodynamic equilibrium (LTE) is assumed in the plasma so that the Boltzmann-Saha distribution for ionization and level populations can be used. In STELLA, no nuclear networks except radioactive decay of nickel-56 to cobalt-56 and to iron-56 are used. To calculate the overall opacity, the code uses 16 species, which include $\mathrm{H}, \mathrm{He}, \mathrm{C}, \mathrm{N}, \mathrm{O}, \mathrm{Ne}, \mathrm{Na}, \mathrm{Mg}, \mathrm{Al}, \mathrm{Si}, \mathrm{S}, \mathrm{Ar}, \mathrm{Ca}$, a sum of stable Fe and radioactive ${ }^{56} \mathrm{Co}$, stable Ni and radioactive ${ }^{56} \mathrm{Ni}$. The energy from such nickel and cobalt radioactive decay is deposited into positrons and gamma-photons. This energy is treated in a one-group transport approximation as suggested by (Swartz, Sutherland \& Harkness 1995).

In STELLA, a Lagrangian co-moving grid is used to solve the 1-dimensional equations for mass, momentum and total energy. Here, the artificial viscosity comprises of the standard von Neumann artificial viscous pressure used for stabilizing solution (von Neumann \& Richtmyer 1950) and a so-called cold artificial viscosity used to smear shocks (Blinnikov et al. 1996). Therefore, STELLA enables one to prop- 
erly compute the shock propagation along the ejecta and the shock breakout event. The coupled equations of radiation hydrodynamics are solved through an implicit high-order predictor-corrector procedure based on the methods of (Brayton et al. 1972) (for more details please look into (Blinnikov et al. 1996) and (Stabrowski 1997)).

\subsection{SuperNova Explosion Code (SNEC)}

SuperNova Explosion Code (SNEC) is an opensource spherically-symmetric Lagrangian radiationhydrodynamic code. It solves for the hydrodynamics and equilibrium-diffusion radiation transport in the envelopes of core-collapse $\mathrm{SNe}$, by considering the recombination effects and the presence of radioactive nickel. After providing a progenitor star model as an input along with a parameter file containing explosion energy, type of explosion, opacity floor and few other parameters, SNEC generates the bolometric light curve, light curves in various observed wavelength bands in the black-body approximation, the photospheric radii, temperature and velocities at different epochs of time, the density profile, luminosity coming out due to radioactive nickel, distribution of initial pressure and many more physical properties.

After the termination of evolution of $24 \mathrm{M}_{\odot}$ on reaching the verge of core-collapse, the outputs of MESA are given as input to STELLA and SNEC after converting into appropriate formats accepted by these two explosion codes. Various explosion parameters and the outcomes are discussed in subsection 3.1 .

\subsection{MOSFIT}

MOSFiT is a Python-based package. It downloads the data sets of various transient from openly available online catalogs, such as, the Open Supernova Catalog ${ }^{15}$, and then generates Monte Carlo ensembles of semi analytical light curve fits to those data sets and their associated Bayesian parameter posteriors, and in the end, on the basis of user's choice, sends the fitting results back to those same catalogs so that the rest of the community can use it. There are a number of models that are currently available in MOSFiT. Few of them are : a) The default model (Nadyozhin 1994), which considers Nickel-cobalt decay as the powering mechanism to the SN light curve. This model is applicable to the type Ia, Ic, PISN and ca-rich supernovae. b) The ic model (Nadyozhin 1994), particularly applicable for type Ic SNe. It takes into account the radioactive decay of Nickel-56 and Cobalt-56 along

\footnotetext{
${ }^{15}$ https://sne.space/
}

with the synchrotron radiations. Like $i c$ model, there is a seperate model available for type Ia SNe events which is known as ia model (Nadyozhin 1994). This model includes typical NiCo decay along with I-band features. c) The csm model (Chatzopoulos et al. 2013; Villar et al. 2017a), which are the interacting CSM-SNe, are applicable to the super luminous SN (SLSN)-II, type IIn SNe. d) The exppow model (Guillochon et al. 2018), which shows an exponential rise and power law decay and is applicable to all type of supernovae. e) The magnetar model (Nicholl et al. 2017a), having Magnetar engine and displaying simple Spectral Energy Distribution (SED). This model is applicable to SLSN-I. Another model, which is applicable to SLSN-I, is the magni model (Nicholl et al. 2017a). This model encorporates the combined effects of Magnetar and the NiCo decay. There is one more model available in MOSFiT which can be used for SLSN-I. It is the slsn model (Nicholl et al. 2017a), which encorporates Magnetar, modified SED and some constraints.

Few more models are also available in MOSFiT, which can be applied to Tidal Disruption events, tde (Mockler at al. 2018), kilonova (Villar et al. 2017b) and $r$-process (Metzger 2017; Villar et al. 2017a) decays. Thus MOSFiT prove to be an important tool to not only SNe but also to some other transient events.

Here in this project, we have tried to fit the light curves of a particular type Ic SN, SN1999ex (Stritzinger et al. 2002). The controls of fitting parameters and the results of the analysis are discussed in sub-section3.2.

\section{4. $S N C O S M O$}

SNCOSMO is a python based tool for SN analysis. It can be used for synthesizing SN spectra and also photometry using the SN models available. There are various SN models or templates available in SNCOSMO, which can be used to synthesize spectra and photometry by varying various parameters such as SN red shift (z), explosion epoch $\left(t_{0}\right)$, host extinction, galactic extinction, dust maps and few more parameters also. It provides various functions which can be used for the fitting and sampling SN model parameters to the user provided photometric data. It provides convenience functions that can be used to read and write the peculiar data formats which are used by various other packages. SNCOSMO has many built-in supernova models that include Hsiao, SNANA, SALT2 and many more. Along with these models, it has also a variety of built-in magnitude systems and band passes also. 
a

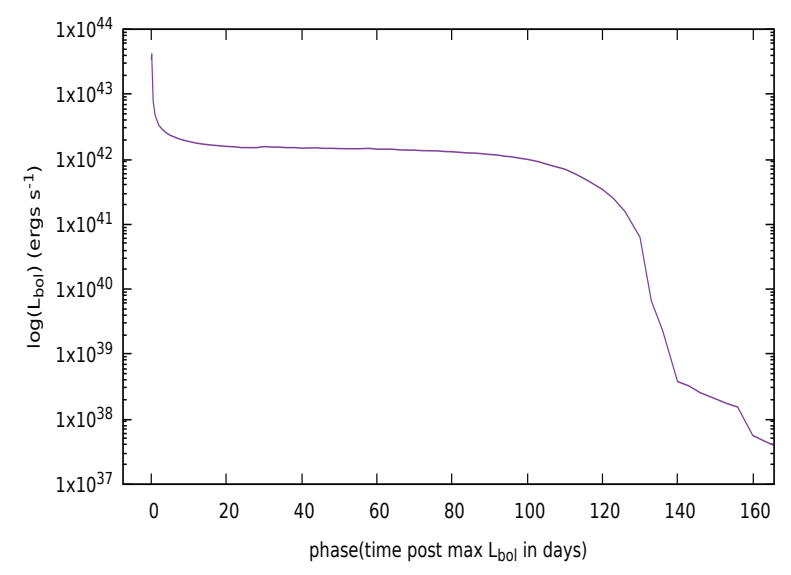

b

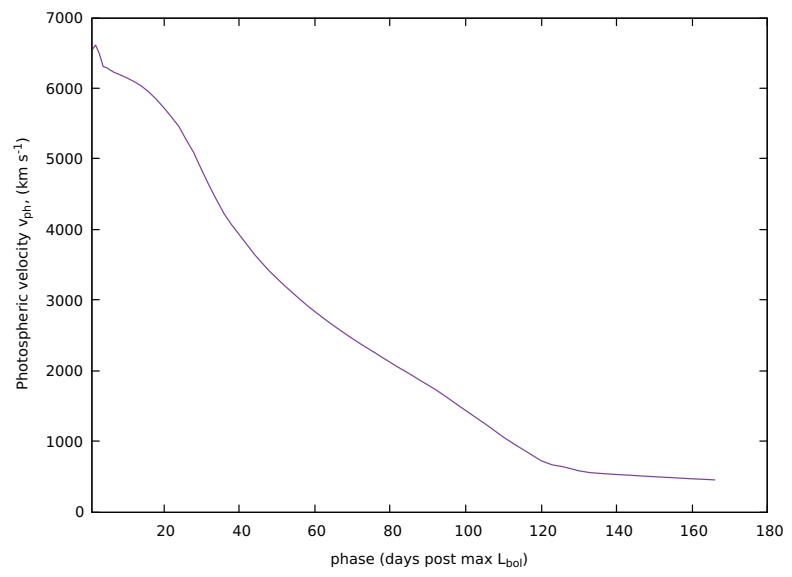

Fig. 3. Left panel shows the bolometric luminosity using STELLA while the right panel shows the evolution of photospheric velocity.
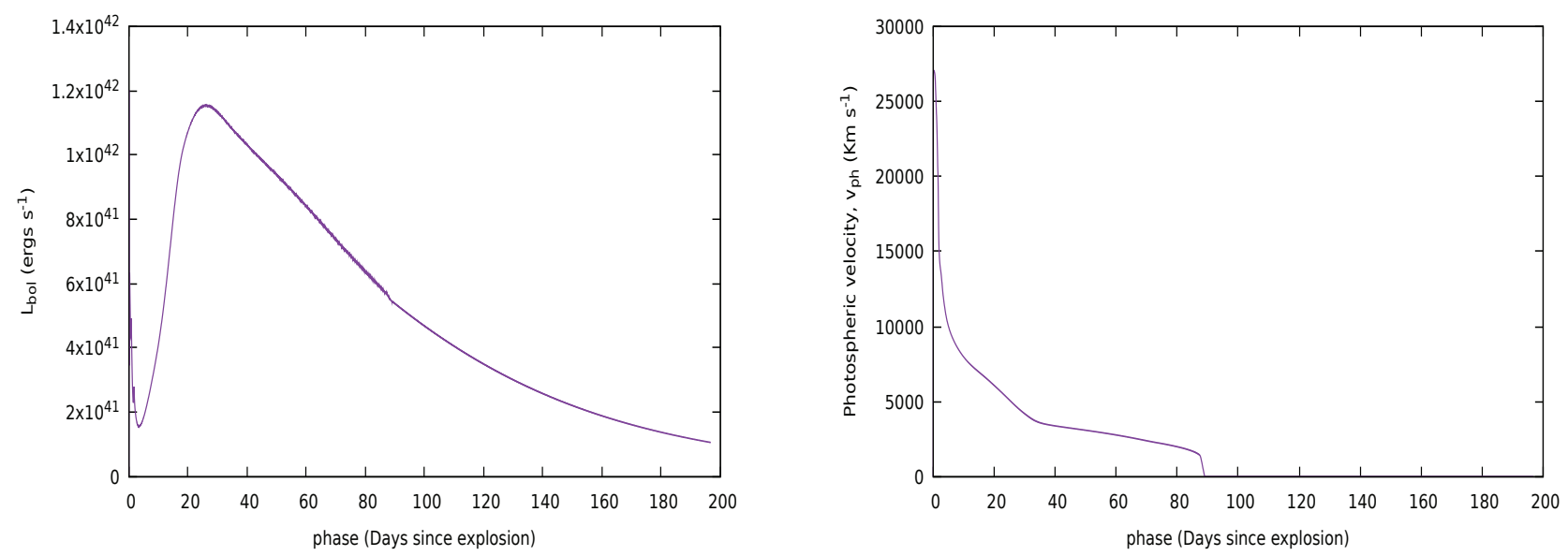

Fig. 4. Left panel shows the observed bolometric luminosity obatined using SNEC and the right panel show the evolution of photospheric velocity.

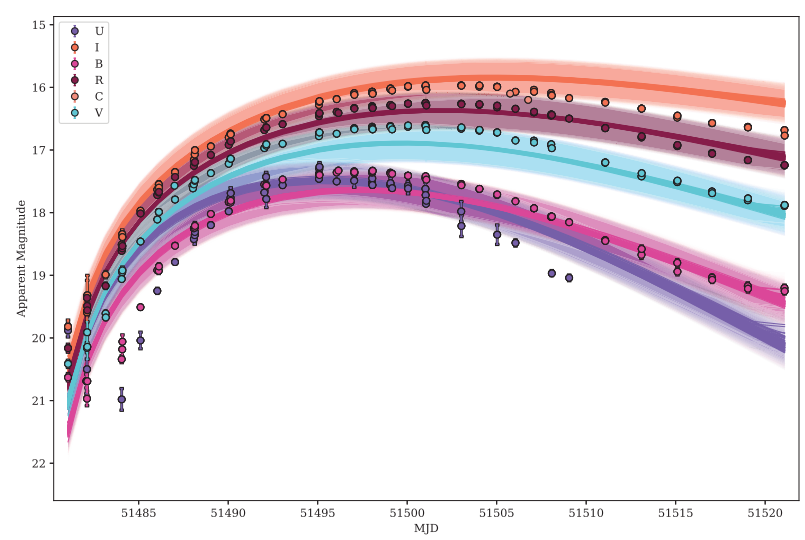

Fig. 5. The result of MOSFiT fitting. Here the U,B,V,R and I bands are fit.
SNCOSMO is extensible. Using a object oriented interface, we can also define new models, new band passes and also magnitude systems. As outputs, SNCOSMO provides the number of $\chi^{2}$ function calls, number of D.O.F in the fit, the $\chi^{2}$ value at minimum, a list of model fit parameters \& there best fit values and finally a list of attributes of the result. Fig.7 shows the results of our fittings. Throughout our analysis, we have used version 2.1.0 of SNCOSMO.

In our analysis, we are trying to reproduce the results as explained in the documentation page ${ }^{16}$ of SNCOSMO by slight modifications in fitting parameters. We have used sample data provided in the same page for our demonstration. The detailed explanation about the fitting parameters and results are discussed in sub-section 3.3.

\footnotetext{
${ }^{16}$ sncosmo.readthedocs.io
} 


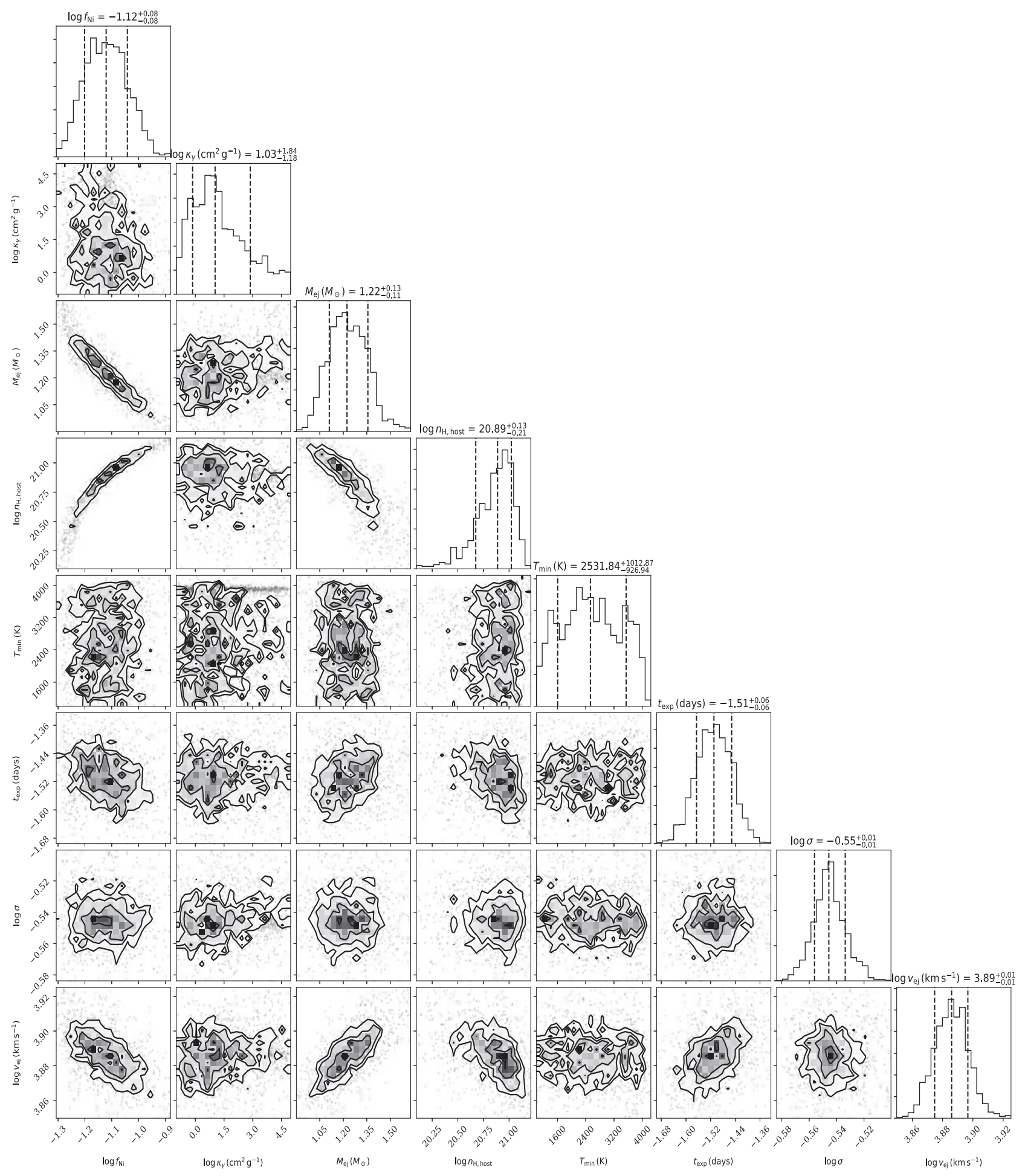

Fig. 6. Corner plot of parameters that were obtained using MOSFiT. $3 \sigma$ levels are shown with vertical dotted lines.

\section{RESULTS}

In this section, we present the outcomes of various analysis in details. We begin with the results obtained using MESA and SNEC, then the results of MOSFiT fittings and finally conclude our result section by discussing the outcomes of SNCOSMO analysis. 

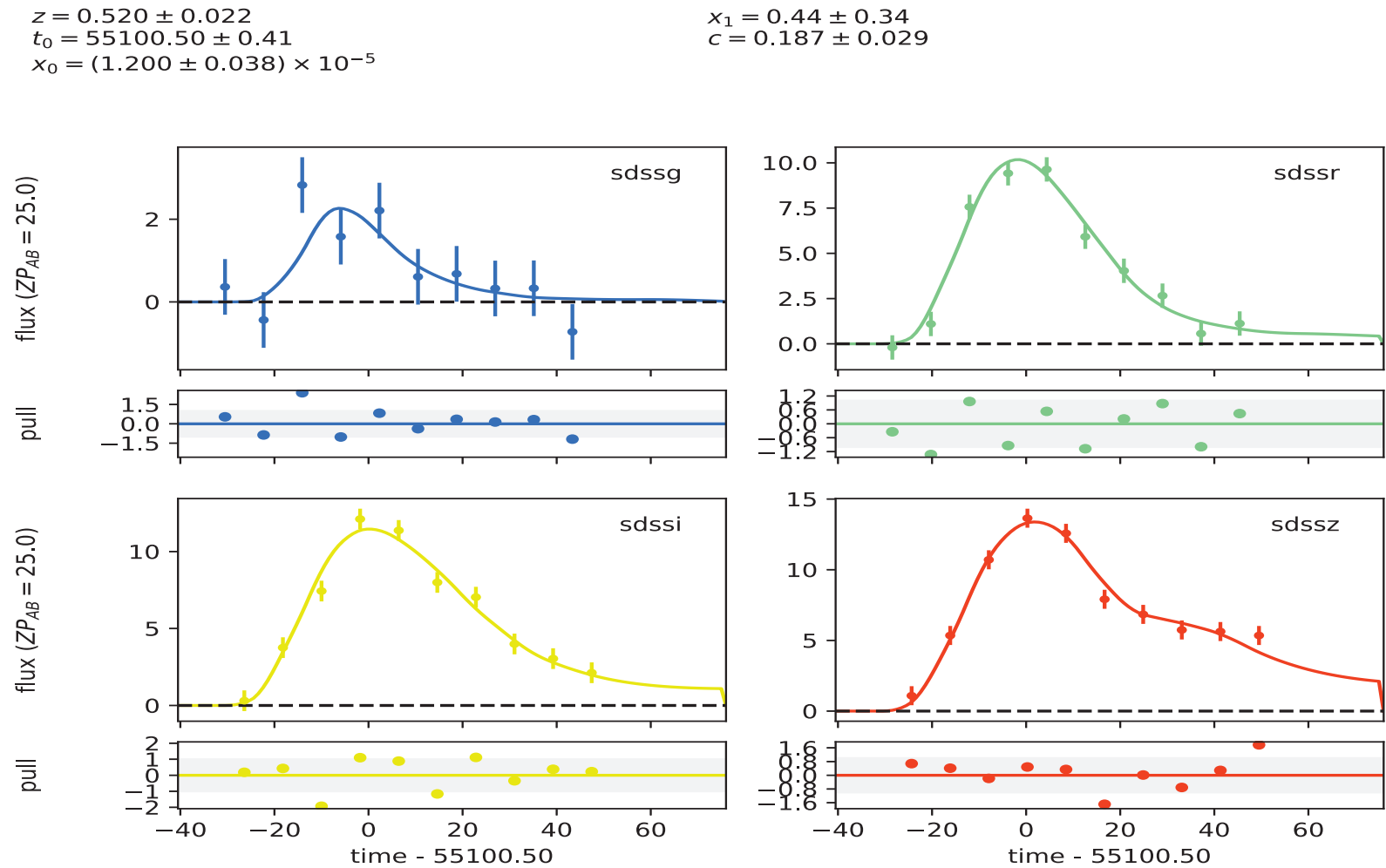

Fig. 7. Result of fitting of salt2 model to the data provided by the SNCOSMO documentation page.

\subsection{MESA \& SNEC analysis}

We took a $24 \mathrm{M}_{\odot}$ star and evolved it through various stages and finally it reaches upto the stage of core-collapse. For this purpose, we used the 25M_pre_ms_to_core_collapse directory of MESA. Fig.2 show the physical condition of our model star during its evolutionary phases after it has passed Si burning phase in its core and approaching a core temperature $(\log \mathrm{T})$ of $9.9 \mathrm{~K}$. The left most panel of fig.2 shows the variation of central density versus central temperature. The top section of mid panel shows the mass fraction of various elements, while the mid section the luminosity corresponding to various processes while the bottom section of mid panel shows the opacity profile of the star. The right-most panel in the same figure the physical parameter variations such as variation in temperature, density, radius etc. To actually explode the star before handing off to STELLA, we first excise the Fe core. For this purpose, we used the final model produced at core-collapse as the initial model in the inlist_infall control file of example_ccsn_IIP directory of MESA. The total energy of explosion is set to $8 \times 10^{50}$ ergs and a negligible trace, $1.0 \times 10^{-15}$ $\mathrm{M}_{\odot}$ of Nickel mass $\left(\mathrm{M}_{N i}\right)$ is used. The outputs of MESA run on example_ccsn_IIP directory include mesa.hyd and mesa.abn files, which are the exact files that are given as the input to STELLA. The outputs of STELLA run are plotted in fig.3. The left panel of fig. 3 shows the variation of bolometric luminosity with time. The bolometric luminosity curve is identical to the conventional type IIP SN bolometric light curve. The maximum bolometric luminosity comes out to be few times $10^{43} \mathrm{ergs} / \mathrm{sec}$. Just few days after the bolometric maximum (nearly at about 10 days), the SN enters the plateau phase. The bolometric luminosity remains constant up to around 90 days, beyond which it falls very rapidly up to a periods of 140 days since bolometric maximum. The right panel of fig. 3 shows the velocity evolution of SN with time. The photospheric velocity evolution also follows the typical trend for a type IIP SN. Initially, the velocity reaches about $6500 \mathrm{~km} \mathrm{~s}^{-1}$, which tend to slowly decreases and attains a constant value of few $100 \mathrm{~km} \mathrm{~s}^{-1}$ beyond a period of 130 days since bolometric maximum.

We also used another explosion code, SNEC, to simulate the explosion. For this purpose, we took the output of final stage of evolution through MESA and converted it into the format as required by SNEC. The explosion in this case is mainly controlled by a parameter file, known as parameters. There, we took the explosion to be of type thermal_bomb. The final energy of bomb is set to be $1.0 \times 10^{51}$. 
we set the gridding to be uniform_in_mass and used the Paczynski equation of state. The nickel mass was taken to be $0.1 \mathrm{M}_{\odot}$. For modelling the opacity, the opacity_floor_envelope and opacity_floor_core were taken to be $0.01 \mathrm{~cm}^{2}$ $\mathrm{g}^{-1}$ and $0.24 \mathrm{~cm}^{2} \mathrm{~g}^{-1}$ respectively. A similar study is going to be published in (Amar et al. 2020a), where we have tried to model the bolometric luminosity and photospheric velocity of a type Ib SN SN2015ap. The work is still in progress and will be published soon anytime in the year 2020 .

The left panel of fig. 4 shows the variation of observed bolometric luminosity curve resulting from SNEC. The bolometric light curve here, resembles much closely the bolometric light curve of a typical type IIL SN. Here the maximum bolometric luminosity turns out to be nearly $1.2 \times 10^{43} \mathrm{ergs} \mathrm{s}^{-1}$, which occurs at around 35 days since explosion. Beyond which, the decline rate becomes almost linear, mimicking the light curve of a type IIL SN.

The right panel of fig. 4 shows the photospheric velocity evolution. Initial velocities are very high, reaching about $28,000 \mathrm{~km} \mathrm{~s}^{-1}$, which shows sharp decline just after few days since explosion and reaches below $5000 \mathrm{~km} \mathrm{~s}^{-1}$ just after 30 days since explosion.

Thus we find that a model having similar initial configurations such as mass metallicity etc. but different explosion mechanism can result in different SNe. The nickel mass present also plays a significant role in determining the shape of light curve and thus determining the SN type.

\subsection{MOSFIT RESULTS}

We selected SN1999ex for our MOSFiT analysis which a type Ic SN. In MOSFiT, we have liberty to select or ignore a particular band for fitting. In the case of SN1999ex, we ignored the clear (C) band data in the fitting process. The fitting parameters are controlled in a parameter file, which is located in the $i c$ sub-directory of models directory of MOSFiT.

In MOSFiT, there are a number of parameter controls available. We provided the ranges of parameters in the form min_value and max_value. For getting satisfactory fittings, we set the min_value and max_value to be $10^{3} \mathrm{~K} \& 10^{5} \mathrm{~K}$ respectively for temperature. As the type Ic SNe results from massive progenitor stars having masses beyond $8 \mathrm{M}_{\odot}$, so, for ejecta mass (mejecta), these values were set to $10^{-2} \mathrm{M}_{\odot} \& 10.0 \mathrm{M}_{\odot}$. Similarly for expanding ejecta velocity, min_value and max_value values were set to be $10^{3} \mathrm{~km} \mathrm{~s}^{-1} \& 10^{5} \mathrm{~km} \mathrm{~s}^{-1}$, since the typical ejecta speed in case of a type Ic SN reaches few 10,000. The values of min_value and max_value for the gamma-ray opacity of the $\mathrm{SN}$ ejecta were set to be $0.1 \mathrm{~cm}^{2} \mathrm{~g}^{-1}$ and $1.0 \times 10^{6} \mathrm{~cm}^{2} \mathrm{~g}^{-1}$. MOSFiT run on this SN event was adjusted to perform 12000 iterations. A much better fit can be achieved if we slightly tune the parameters and increase the number of iterations or if we let MOSFiT perform iterations until it converges itself but it is extremely time consuming, so we are satisfied with fittings obtained after performing 12000 iterations. The corresponding fitting results are shown in fig.5. The best fitting parameters are shown in a corner plot in fig. 6 with the $3 \sigma$ levels indicated.

\subsection{SNCOSMO RESULTS}

In this section, we have the results of use of SNCOSMO for SN analysis. The fitting process can be described here in three steps :

a) Loading of the photometric data. Here in our case, we have loaded some photometric data, which is already available in SNCOSMO.

b) Creating the model by loading the template.

c) Run the fit. After loading the photometric data and creating the model, we can run the fitting process. It takes few seconds only.

To fit the sample data, we used the SALT2 model as template. There are various fitting parameters in this model. Few of them are explosion epoch $\left(t_{0}\right)$, the SN red shift $(\mathrm{z})$, stretch factor $\left(x_{1}\right)$ of the light curve and many more. We can provide the bounds over the fitting parameters. As an example, we have provided an inner and outer bond of $0.52 \& 0.72$ over red shift, and similarly we can set bounds to other fitting parameters also. There was no need to include the dust map, as we could get very nice fitting without it. With the given bound to the red shift, we could find that the sample SN has a red shift of $0.520 \pm 0.022$, an explosion epoch $\left(t_{0}\right)$ of MJD $55100.50 \pm 0.41$ and a stretch factor $\left(x_{1}\right)$ of $0.44 \pm 0.34$.

\section{SUMMARY AND CONCLUSIONS}

We found that robust analysis of photometric and spectroscopic data of various cosmic energetic transients have a great potential to explore underlying physics using various publicly available tools like MESA, SNEC, MOSFiT, SNCOSMO etc. For example, MESA along with 1D explosion model called SNEC could be used to constrain possible progenitors and their basic physical parameters governing the explosion. From our present results discussed in Section 3.1, we could see that with the help of MESA and SNEC, reliable models of the possible progenitors of core-collapse $\mathrm{SNe}$ and the underlying physical models of explosions could be reproduced, showing 
usefullness in $\mathrm{SN}$ analysis. Another available tool as discussed in Section 3.2 called MOSFiT is used for deriving various physical parameters responsible for the explosion of various types of SNe. These physical parameters include synthesized Nickel mass, opacity, SN temperature and many more parameters. Hence, MOSFiT prove to be an important tool to robustly determine these physical parameters. In Section 3.3, we employed SNCOSMO and showed that it could also be used for template model fitting to various types of SNe to constrain their nature of explosion by varying parameters of template such as the light curve stretch factor $\left(x_{0}\right)$, the red-shift $(\mathrm{z})$ of the $\mathrm{SN}$, the explosion epoch $\left(t_{0}\right)$ etc. Hence, we conclude that robust analysis with the help of such analysis tools could be performed adding value towards ongoing efforts to understand these explosions better.

Acknowledgments: AA, SBP and RG acknowledge BRICS grant DST/IMRCD/BRICS/Pilotcall1/ProFCheap/201 7(G) for the financial support to perform part of the present analysis. This work has been partially supported by the Spanish Science Ministry "Centro de Excelencia Severo Ochoa" Program under grant SEV-2017-0709.

\section{REFERENCES}

Aryan, A. et al., 2020, In preparation

Barbary, K., Barclay, T., Biswas, R., et al. 2016, Astrophysics Source Code Library, record ascl:1611.017

Bersten, M. C., Folatelli, G., Garcia, F., et al. 2018, Natur, 554, 497

Blinnikov, S. I. \& Panov, I. V. 1996, AstL, 22, 39

Blinnikov, S. I., Eastman, R., Bartunov, O. S., Popolitov, V. A., \& Woosley, S. E. 1998, ApJ, 496, 454

Blinnikov, S., Lundqvist, P., Bartunov, O., Nomoto, K., \& Iwamoto, K. 2000, ApJ, 532, 1132

Blinnikov, S. I., Röpke, F. K., Sorokina, E. I., et al. 2006, A\&A, 453, 229

Brayton, R. K., Gustavson, F. G., \& Hatchel, G. D. 1972, Proc. IEEE
Castro-Tirado, A. J. 2010, Acta Polytechnica, 51, 16

Chatzopoulos, E., Wheeler, J. C., Vinko, J., Horvath, Z. L., \& Nagy, A. 2013, ApJ, 773, 76

Eastman, R. G. \& Pinto, P. A. 1993, ApJ, 412, 731

Filippenko, A. V. 1997, ARA\&A, 35, 309

Guillochon, J., Nicholl, M., Villar, V. A., et al. 2018, ApJS, 236, 6

Henyey, L., Vardya, M. S., \& Bodenheimer, P. 1965, ApJ, 142,841

Herwig, F. 2000, A\&A, 360, 952

Kurucz R. L. \& Bell, B. 1995, Kurucz CD-ROM, Cambridge, MA: Smithsonian Astrophysical Observatory

Metzger, B. D. 2017, LRR, 20, 3

Mockler, B., Guillochon, J., \& Ramirez-Ruiz, E. 2018, arXiv:1801.08221

Morozova, V., Piro, A. L., Renzo, M., et al. 2015, ApJ, 814,63

Nadyozhin, D. K. 1994, ApJS, 92, 527

Nicholl, M., Guillochon, J., \& Berger, E. 2017a, ApJ, 850, 55

Nicholl, M., Williams, P. K. G., Berger, E., et al. 2017b, ApJ, 843, 84

Paxton, B., Bildsten, L., Dotter, A., et al. 2011, ApJS, 192, 3

Paxton, B., Cantiello, M., Arras, P., et al. 2013, ApJS, 208, 4

Paxton, B., Marchant, P., Schwab, J., et al. 2015, ApJS, 220, 15

Paxton, B., Schwab, J., Bauer, E. B., et al. 2018, ApJS, 234,34

Smartt, S. J. 2009, ARA\&A, 47, 63

Pandey, S. B. 2016, RMxAC, 48, 83

Stabrowski, M. M. 1997, Simul. Modelling Pract. Theory, 5,333

Stritzinger, M., Hamuy, M., Suntzeff, N. B., et al. 2002, AJ, 124, 2100

Swartz, D. A., Sutherland, P. G., \& Harkness, R. P. 1995, ApJ, 446, 766

Verner, D. A., Verner, E. M., \& Ferland, G. J. 1996, At. Data Nucl. Data Tables, 64, 1

Villar, V. A., Berger, E., Metzger, B. D., \& Guillochon, J. 2017a, ApJ, 849, 70

Villar, V. A., Guillochon, J., Berger, E., et al. 2017a, ApJL, 851, 21

von Neumann, J. \& Richtmyer, R. D. 1950, JAP, 21, 232 